\title{
ANÁLISE DO TEMPO DE ALINHAMENTO EM SISTEMAS DE NAVEGAÇÃO INERCIAL SOLIDÁRIOS
}

\author{
Felipe Oliveira e Silva*, Waldemar de Castro leite Filho* \\ * Instituto de Aeronáutica e Espaço - Divisão de Sistemas Espaciais \\ Praça Marechal Eduardo Gomes, 50 - Vila das Acácias \\ São José dos Campos, SP, Brasil \\ Emails: felipefos@iae.cta.br, waldemarwclf@iae.cta.br
}

\begin{abstract}
Resumo- Neste artigo é apresentada uma metodologia de auto-alinhamento usualmente empregada em sistemas de navegação inercial solidários (SINS) e sua correspondente análise de erros. É demonstrado que, com excessão do alinhamento em azimute, esta metodologia é influenciada basicamente pelas incertezas atreladas às leituras dos acelerômetros. Uma análise estatística utilizando o desvio padrão de Allan é realizada e permite se estimar analiticamente o tempo necessário para se obter uma determinada classe de precisão no alinhamento em azimute. Este resultado é comparado com resultados experimentais obtidos a partir de um SINS de alta precisão, demonstrando que as incertezas provenientes do giroscópio apontado para leste constituem o fator preponderante na determinação do tempo de alinhamento em azimute, que por sua vez, determina o tempo e a precisão final do alinhamento em um SINS.
\end{abstract}

Palavras-chave- Navegação e auto-localização, Sistema de navegação inercial solidário, Auto-alinhamento, Tempo de alinhamento.

\begin{abstract}
This paper presents a self-alignment methodology commonly used in strapdown inertial navigation systems (SINS) and its corresponding error analysis. It is shown that, except for azimuth alignment, such a methodology is primarily influenced by the accelerometers uncertainties. A statistical analysis using the Allan standard deviation is performed and allows to analytically estimate the time required to obtain a particular accuracy class on azimuth alignment. This result is compared with experimental results obtained from a high precision SINS, demonstrating the east gyroscope uncertainties are the predominant factor in determining the azimuth alignment time, which in turn determines the final alignment time and accuracy on a SINS.
\end{abstract}

Keywords - Navigation and self-location, Strapdown inertial navigation system, Self-alignment, Alignment time.

\section{Introdução}

A determinação da posição, velocidade, e orientação de um corpo por meio de sistemas de navegação inercial solidários (SINS) só é possível através da integração numérica das variáveis medidas pelos acelerômetros e giroscópios a cada instante de amostragem. Como todo e qualquer processo de integração, para que se chegue a valores corretos para as variáveis integradas, é necessário que as condições iniciais da posição, velocidade, e orientação sejam fornecidas previamente. A este processo dá-se o nome de inicialização (Jekeli, 2000).

Para grande parte das aplicações atuais (Farrel and Barth, 1999), a inicialização só pode ser realizada em condições ditas estacionárias. Em tais condições, a menos de perturbações externas causadas por vibrações mecânicas e rajadas de vento, a posição e a velocidade iniciais do veículo são conhecidas com precisão e estão disponíveis $a$ priori (a velocidade é considerada nula, uma vez que o veículo é suposto estar parado, e a posição pode ser determinada por meios externos, via, por exemplo, GPS).

A despeito das condições iniciais para a posição e a velocidade do corpo, a orientação inicial do mesmo não é tão facilmente determinada, e no caso de veículos aeroespaciais, (foco de aplicação deste artigo), é de suma importância que o SINS seja capaz de determinar a orientação inicial do mesmo sem nenhum auxílio externo (Titterton and Weston, 2004). Ao processo de inicialização da orientação do veículo, dá-se o nome de alinhamento. No caso desta inicialização ser realizada sem nenhum auxílio externo, o processo é chamado auto-alinhamento (Savage, 2007).

O principal objetivo deste artigo é analisar o compromisso existente entre a acurácia do procedimento de auto-alinhamento e o respectivo tempo de alinhamento necessário para se obter uma determinada classe de precisão. Na Seção 2 deste artigo são apresentadas uma metodologia de autoalinhamento usualmente referida na literatura e sua respectiva análise de erros. A Seção 3 caracteriza as principais fontes de erros existentes em giroscópios e sua influência no tempo de alinhamento. Na Seção 4, uma análise estatística é realizada sobre os dados provenientes de um SINS de alta precisão, e os resultados experimentais possibilitam uma comparação entre o tempo de alinhamento e a acurácia obtida. As conclusões são apresentadas na Seção 5.

\section{Auto-alinhamento grosseiro}

\subsection{Dedução analítica}

Uma das metodologias de auto-alinhamento mais frequentemente empregadas em SINS's (Jiang, 1998) baseia-se na medição de três vetores não- 
colineares provenientes da combinação dos vetores gravidade $\vec{g}$ e velocidade angular da terra $\vec{\omega}_{I E}$, a saber, a tríade $\left(\vec{g}, \vec{g} \times \vec{\omega}_{I E},\left(\vec{g} \times \vec{\omega}_{I E}\right) \times \vec{g}\right)$, cujas componentes são bem conhecidas no triedro de navegação, isto é:

$$
\begin{aligned}
\vec{g}^{N} & =\left[\begin{array}{c}
-g \\
0 \\
0
\end{array}\right] \\
\left(\vec{g} \times \vec{\omega}_{I E}\right)^{N} & =\left[\begin{array}{c}
0 \\
g \omega_{I E} \cos L \\
0
\end{array}\right] \\
\left(\left(\vec{g} \times \vec{\omega}_{I E}\right) \times \vec{g}\right)^{N} & =\left[\begin{array}{c}
0 \\
0 \\
g^{2} \omega_{I E} \cos L
\end{array}\right]
\end{aligned}
$$

onde $g$ e $\omega_{I E}$ representam a magnitude dos vetores gravidade e velocidade angular da terra, respectivamente, $L$ é a latitude do corpo e o índice ${ }^{N}$ indica que os vetores estão representados no triedro de navegação. O triedro de navegação é aqui definido com seus eixos $x_{N}, y_{N}$ e $z_{N}$ apontando, respectivamente, para as direções vertical local, leste e norte (configuração $U E N$ - up, east, north) e o veículo é suposto estar no hemisfério sul.

Estes mesmos vetores podem ser representados no triedro do corpo, e especialmente para o caso do alinhamento estacionário, são definidos como:

$$
\begin{gathered}
\vec{g}^{B}=\left[\begin{array}{l}
-f_{x} \\
-f_{y} \\
-f_{z}
\end{array}\right] \\
\left(\vec{g} \times \vec{\omega}_{I E}\right)^{B}=\left[\begin{array}{c}
f_{z} \omega_{y}-f_{y} \omega_{z} \\
f_{x} \omega_{z}-f_{z} \omega_{x} \\
f_{y} \omega_{x}-f_{x} \omega_{y}
\end{array}\right] \\
\left(\left(\vec{g} \times \vec{\omega}_{I E}\right) \times \vec{g}\right)^{B}= \\
=\left[\begin{array}{c}
f_{z}^{2} \omega_{x}-f_{x} f_{z} \omega_{z}-f_{x} f_{y} \omega_{y}+f_{y}^{2} \omega_{x} \\
f_{x}^{2} \omega_{y}-f_{x} f_{y} \omega_{x}-f_{y} f_{z} \omega_{z}+f_{z}^{2} \omega_{y} \\
f_{y}^{2} \omega_{z}-f_{y} f_{z} \omega_{y}-f_{x} f_{z} \omega_{x}+f_{x}^{2} \omega_{z}
\end{array}\right]
\end{gathered}
$$

onde $f_{x}, f_{y}$ e $f_{z}$ são as forças específicas medidas pelos acelerômetros e $\omega_{x}, \omega_{y}$ e $\omega_{z}$ são as velocidades angulares medidas pelos giroscópios, nos respectivos eixos $x, y$ e $z$. O índice ${ }^{B}$ indica que os vetores estão representados no triedro do corpo.

A representação dos vetores acima definidos segue a seguinte relação:

$$
\begin{gathered}
\vec{g}^{N}=C_{B}^{N} \vec{g}^{B} \\
\left(\vec{g} \times \vec{\omega}_{I E}\right)^{N}=C_{B}^{N}\left(\vec{g} \times \vec{\omega}_{I E}\right)^{B} \\
\left(\left(\vec{g} \times \vec{\omega}_{I E}\right) \times \vec{g}\right)^{N}=C_{B}^{N}\left(\left(\vec{g} \times \vec{\omega}_{I E}\right) \times \vec{g}\right)^{B}
\end{gathered}
$$

onde $C_{B}^{N}$ é a matriz de transformação de coordenadas (DCM) que relaciona o triedro do corpo ao triedro de navegação, e que, portanto, serve para caracterizar o alinhamento inicial do veículo.
Definindo-se:

$$
\begin{aligned}
& N=\left[\begin{array}{c}
\vec{g}^{N} \\
\left(\vec{g} \times \vec{\omega}_{I E}\right)^{N} \\
\left(\left(\vec{g} \times \vec{\omega}_{I E}\right) \times \vec{g}\right)^{N}
\end{array}\right]^{T} \\
& B=\left[\begin{array}{c}
\vec{g}^{B} \\
\left(\vec{g} \times \vec{\omega}_{I E}\right)^{B} \\
\left(\left(\vec{g} \times \vec{\omega}_{I E}\right) \times \vec{g}\right)^{B}
\end{array}\right]^{T}
\end{aligned}
$$

Temos:

$$
N=C_{B}^{N} B
$$

Como, por definição, $C_{B}^{N}=\left(C_{N}^{B}\right)^{T}$ :

$$
C_{B}^{N}=\left(N^{-1}\right)^{T} B^{T}
$$

Efetuando as operações acima, chega-se a:

$$
C_{B}^{N}=\left[\begin{array}{lll}
c_{11} & c_{12} & c_{13} \\
c_{21} & c_{22} & c_{23} \\
c_{31} & c_{32} & c_{33}
\end{array}\right]
$$

onde:

$$
\begin{gathered}
c_{11}=\frac{f_{x}}{g} \\
c_{12}=\frac{f_{y}}{g} \\
c_{13}=\frac{f_{z}}{g} \\
c_{21}=\frac{f_{z} \omega_{y}-f_{y} \omega_{z}}{g \omega_{I E} \cos L} \\
c_{22}=\frac{f_{x} \omega_{z}-f_{z} \omega_{x}}{g \omega_{I E} \cos L} \\
c_{23}=\frac{f_{y} \omega_{x}-f_{x} \omega_{y}}{g \omega_{I E} \cos L} \\
c_{31}=\frac{f_{z}^{2} \omega_{x}-f_{x} f_{z} \omega_{z}-f_{x} f_{y} \omega_{y}+f_{y}^{2} \omega_{x}}{g^{2} \omega_{I E} \cos L} \\
c_{32}=\frac{f_{x}^{2} \omega_{y}-f_{x} f_{y} \omega_{x}-f_{y} f_{z} \omega_{z}+f_{z}^{2} \omega_{y}}{g^{2} \omega_{I E} \cos L} \\
c_{33}=\frac{f_{y}^{2} \omega_{z}-f_{y} f_{z} \omega_{y}-f_{x} f_{z} \omega_{x}+f_{x}^{2} \omega_{z}}{g^{2} \omega_{I E} \cos L}
\end{gathered}
$$

Outra forma mais intuitiva de se caracterizar o alinhamento inicial de um SINS é através dos ângulos de Euler. Os ângulos de Euler são coordenadas angulares definidas por uma seqüência de três rotações sobre os eixos coordenados do triedro de navegação. No caso de veículos aeroespaciais, uma seqüência usual de rotações é a seguinte: rotação de um ângulo $\theta$ sobre o eixo $y_{N}$ (ângulo de arfagem ou pitch); rotação de um ângulo $\psi$ sobre o novo eixo $z_{N}^{\prime}$ (ângulo de guinada, yaw ou heading) e, finalmente, rotação de um ângulo $\phi$ sobre o novo eixo $x_{N}^{\prime \prime}$ (ângulo de rolagem ou roll).

Para a sequência de rotações acima descrita, e valendo-se da ferramenta piograma, apresentada 
em (Pio, 1966) pode-se exprimir os ângulos de Euler em função da DCM:

$$
\begin{gathered}
\theta=\tan ^{-1} \frac{-c_{13}}{c_{11}} \\
\psi=\sin ^{-1} c_{12} \\
\phi=\tan ^{-1} \frac{-c_{32}}{c_{22}}
\end{gathered}
$$

Substituindo (15), (16), (17), (19) e (22) em (24), (25) e (26), tem-se:

$$
\begin{gathered}
\theta=\tan ^{-1} \frac{-f_{z}}{f_{x}} \\
\psi=\sin ^{-1} \frac{f_{y}}{g} \\
\phi=\tan ^{-1} \frac{f_{x}^{2} \omega_{y}-f_{x} f_{y} \omega_{x}-f_{y} f_{z} \omega_{z}+f_{z}^{2} \omega_{y}}{g\left(f_{z} \omega_{x}-f_{x} \omega_{z}\right)}
\end{gathered}
$$

Assim, utilizando-se (27), (28) e (29), o alinhamento inicial do veículo em termos de ângulos de Euler pode ser determinado diretamente a partir das leituras dos sensores inerciais e do valor previamente conhecido para $g$, independentemente da latitude $L$ do veículo.

\subsection{Análise de erros}

Embora a abordagem analítica apresentada na Seção 2.1 esteja conceitualmente correta, o valor exato da matriz de transformação de coordenadas $C_{B}^{N}$ não pode ser determinado na prática. Isto se deve fundalmentalmente à existência de ruídos e perturbações externas atreladas às leituras dos sensores inerciais e às pequenas movimentações do veículo. O que se obtêm na prática, portanto, é uma estimativa da $C_{B}^{N}$, representada por $\hat{C}_{B}^{N}$, a qual pode ser genericamente equacionada como (Savage, 2007):

$$
\hat{C}_{B}^{N}=(I+E) C_{B}^{N}
$$

Na equação acima, $E$ é a matriz que contem os erros da $\hat{C}_{B}^{N}$, a qual, desprezando-se eventuais erros de ortonormalidade, pode ser representada por:

$$
E=\left[\begin{array}{ccc}
0 & \epsilon_{z} & -\epsilon_{y} \\
-\epsilon_{z} & 0 & \epsilon_{x} \\
\epsilon_{y} & -\epsilon_{x} & 0
\end{array}\right]
$$

onde os elementos $\epsilon_{x}, \epsilon_{y}$ e $\epsilon_{z}$ são os componentes do chamado vetor de Euler de desalinhamento $\vec{\epsilon}$, que pode ser entendido como sendo o vetor através do qual os eixos coordenados do triedro do corpo devem ser rotacionados para coincidirem com o triedro de navegação, sendo a magnitude $\epsilon$ da rotação dada pela própria magnitude do vetor de desalinhamento, isto é:

$$
\epsilon=\sqrt{\epsilon_{x}^{2}+\epsilon_{y}^{2}+\epsilon_{z}^{2}}
$$

Para o caso de pequenos vetores de desalinhamento, a ordem das rotações em termos de ângulos de Euler é insignificante. Assim, pode-se aproximar: $\epsilon_{x} \approx \delta \phi, \epsilon_{y} \approx \delta \theta$ e $\epsilon_{z} \approx \delta \psi$, onde $\delta \theta, \delta \psi$ e $\delta \phi$ são os erros nos ângulos de Euler calculados em (27) a (29). Assim, tem-se:

$$
E \approx\left[\begin{array}{ccc}
0 & \delta \psi & -\delta \theta \\
-\delta \psi & 0 & \delta \phi \\
\delta \theta & -\delta \phi & 0
\end{array}\right]
$$

Para o caso específico do auto-alinhamento estacionário, pode-se considerar que os erros na determinação da $C_{B}^{N}$ advêm principalmente das incertezas dos sensores inerciais, e assim, pode-se reescrever (13) da seguinte forma:

$$
\hat{C}_{B}^{N}=\left(N^{-1}\right)^{T} \hat{B}^{T}
$$

onde:

$$
\hat{B}=B+\delta B
$$

e:

$$
\delta B=\left[\begin{array}{ccc}
-\delta f_{x} & \delta v_{x} & \delta t_{x} \\
-\delta f_{y} & \delta v_{y} & \delta t_{y} \\
-\delta f_{z} & \delta v_{z} & \delta t_{z}
\end{array}\right]
$$

Nas equações acima, $\delta f_{x}, \delta f_{y}, \delta f_{z}, \delta \omega_{x}, \delta \omega_{y} \mathrm{e}$ $\delta \omega_{z}$ representam os erros nas leituras dos acelerômetros e giroscópios e $\delta v_{x}, \delta v_{y}, \delta v_{z}, \delta t_{x}, \delta t_{y}$ e $\delta t_{z}$ são obtidos pela aplicação da fórmula de propagação de erros em (5) e (6), respectivamente.

Substituindo (35) em (34), obtem-se:

$$
\hat{C}_{B}^{N}=\left(I+\left(N^{-1}\right)^{T} \delta B^{T} C_{N}^{B}\right) C_{B}^{N}
$$

Comparando (30) com (37), conclui-se que:

$$
E=\left(N^{-1}\right)^{T} \delta B^{T} C_{N}^{B}
$$

Infelizmente, para uma escolha arbitrária da $C_{B}^{N},(38)$ não é facilmente interpretável (Britting, 1971), e uma solução particularmente simples só é obtida considerando-se um alinhamento perfeito entre os triedros do corpo e de navegação, isto é $C_{B}^{N}=I$. Neste caso:

$$
E=\left(N^{-1}\right)^{T} \delta B^{T}
$$

e:

$$
\begin{gathered}
\delta v_{x}=-\delta f_{y} \omega_{I E} \cos L \\
\delta v_{y}=\delta f_{x} \omega_{I E} \cos L-\delta f_{z} \omega_{I E} \sin L+\delta \omega_{z} g \\
\delta v_{z}=\delta f_{y} \omega_{I E} \sin L-\delta \omega_{y} g \\
\delta t_{x}=-g \omega_{I E} \cos L \delta f_{z} \\
\delta t_{y}=-g \omega_{I E} \sin L \delta f_{y}+g^{2} \delta \omega_{y} \\
\delta t_{z}=g \omega_{I E} \cos L \delta f_{x}-g \omega_{I E} \sin L \delta f_{z}+g^{2} \delta \omega_{z}
\end{gathered}
$$

Substituindo-se (40) a (45) em (36), (10) em (39), e o resultado em (33), obtem-se:

$$
\delta \theta=-\frac{\delta f_{z}}{g}
$$




$$
\begin{gathered}
\delta \psi=\frac{\delta f_{y}}{g} \\
\delta \phi=\frac{\delta f_{y} \tan L}{g}-\frac{\delta \omega_{y}}{\omega_{I E} \cos L}
\end{gathered}
$$

Analisando-se (46) a (48), observa-se que os erros no cálculo dos ângulos de Euler advêm basicamente dos acelerômetros. A excessão é o ângulo de rolagem (correspondente ao alinhamento em azimute), que depende também das incertezas atreladas ao giroscópio apontado para o leste. Como será mostrado na próxima Seção, os erros provenientes dos giroscópios exercem uma grande influência na acurácia do alinhamento em azimute, e serão o fator preponderante na determinação do tempo de alinhamento total de um SINS.

\section{Tempo de alinhamento em azimute}

\subsection{Randow noise em giroscópios}

Dentre os diversos componentes de erros que contribuem para a incerteza final dos giroscópios (bias, erros de quantização, deriva térmica, erros de fator de escala, erros de desalinhamento mecânico entre os eixos dos giroscópios, etc), o que mais compromete o procedimento de auto-alinhamento de um SINS é o randow noise (Titterton and Weston, 2004).

O randow noise, também conhecido como ruído branco, pode ser entendido como uma variação de alta frequência do sinal de saída dos giroscópios, quando estes estão em repouso, perfeitamente nivelados e não submetidos a nenhum tipo de translação. A integração deste tipo de ruído é usualmente referida na literatura pelo nome de randow walk angular, e define um tipo de erro que cresce proporcionalmente à raiz quadrada do tempo de alinhamento.

Para se reduzir o erro causado pelo randow noise dos giroscópios na estimativa do alinhamento em azimute, (Titterton and Weston, 2004) mostra que as leituras dos sensores inerciais devem ser acumuladas durante um intervalo de tempo $T$ e depois convertidas num valor médio, para então serem utilizadas nos cálculos. Este tempo $T$, chamado de tempo de alinhamento em azimute é calculado como:

$$
T=\left(\frac{r w_{y}}{\delta \phi \omega_{I E} \cos L} \frac{180}{\pi}\right)^{2}
$$

onde $\delta \phi$ é o já definido erro esperado para o alinhamento em azimute e $r w_{y}$ é o randow walk angular do giroscópio apontado para a direção leste, cujo valor, dado em ${ }^{\circ} / \sqrt{h}$, pode ser estimado através de uma técnica estatística conhecida como desvio padrão de Allan (Han et al., 2009).

\subsection{Desvio padrão de Allan}

O desvio padrão de Allan ( $a d$ ) é uma técnica de análise no domínio do tempo originalmente desen- volvida para se caracterizar ruídos e estabilidade em sistemas de relógio GPS. Esta técnica, entretanto, pode ser aplicada a qualquer sinal sob o qual se deseja determinar ruídos de processos ocultos.

Para uma grande massa de dados, o desvio padrão de Allan pode ser determinado dividindo-se a sequência original destes dados em $n$ partições de comprimento temporal $\tau(n \geq 9)$, e extraindo-se, em seguida, o valor médio de cada partição, isto é, $\bar{x}_{1}(\tau), \bar{x}_{2}(\tau), \bar{x}_{3}(\tau), \ldots, \bar{x}_{n}(\tau)$. A partir dos valores médios de cada partição, o desvio padrão de Allan é calculado como:

$$
a d(\tau)=\sqrt{\frac{1}{2(n-1)} \sum\left(\bar{x}_{n}(\tau)-\bar{x}_{n-1}(\tau)\right)^{2}}
$$

Plotando-se o desvio padrão de Allan em função de $\tau$ num gráfico bidimensional com ambas as escalas logarítmicas, observa-se que os diferentes tipos de processos aleatórios produzem curvas com diferentes gradientes de inclinação (Han et al., 2009). Uma vez identificado o tipo de processo aleatório, é possível obter seu valor numérico diretamente por análise gráfica. Para o caso específico dos giroscópios, o valor do randow walk angular é obtido pela interseção da curva do desvio padrão de Allan com a linha perpenticular ao eixo das ordenadas no tempo de partição $\tau=1$.

Uma vez estimado o valor do randow walk angular do giroscópio apontado para a leste, podese utilzar (49), para se estimar o tempo necessário para a obtençao de uma determinada classe de precisão no alinhamento em azimute.

\section{Resultados experimentais}

\subsection{Cálculo do tempo de alinhamento}

De modo a se analisar a relação entre a acurácia e o tempo de alinhamento para o procedimento de auto-alinhamento apresentado na Seção 2, foi realizado um ensaio experimental estático em um SINS de alta precisão (tecnologia $R L G$ ring laser gyroscope).

O procedimento consistiu em se alinhar o SINS utilizando uma mesa rotativa de três eixos, de forma que o triedro do corpo se aproximasse o máximo possível do triedro de navegação, isto é, $C_{B}^{N} \approx I$, tornando válida a aproximação descrita em (33). Em seguida, leituras dos acelerômetros e giroscópios foram tomadas a uma taxa de amostragem de $100 \mathrm{~Hz}$ durante um período de aproximadamente 16 horas.

Uma análise estatística foi realizada sobre as saídas dos sensores, no intuito de se determinar o desvio padrão dos dados e os seus respectivos valores médios verdadeiros, os quais, segundo (Vuolo, 1992), constituem a melhor estimativa para os valores verdadeiros das grandezas medi- 
das, os quais são, por definição, desconhecidos. Os resultados são apresentados na Tabela 1 .

Tabela 1: Sinais provenientes dos sensores inerciais.

\begin{tabular}{c|cc} 
& $\begin{array}{c}\text { Valor médio } \\
\text { verdadeiro }\end{array}$ & $\begin{array}{c}\text { Desvio } \\
\text { padrão }\end{array}$ \\
\hline$f_{x}\left[\mathrm{~m} / \mathrm{s}^{2}\right]$ & 9.7864 & 0.0244 \\
$f_{y}\left[\mathrm{~m} / \mathrm{s}^{2}\right]$ & 0.0112 & 0.0093 \\
$f_{z}\left[\mathrm{~m} / \mathrm{s}^{2}\right]$ & -0.0094 & 0.0086 \\
$\omega_{x}[\mathrm{rad} / \mathrm{s}]$ & $-2.8438 \times 10^{-5}$ & $4.5099 \times 10^{-5}$ \\
$\omega_{y}[\mathrm{rad} / \mathrm{s}]$ & $4.3219 \times 10^{-7}$ & $5.2086 \times 10^{-5}$ \\
$\omega_{z}[\mathrm{rad} / \mathrm{s}]$ & $6.6829 \times 10^{-5}$ & $4.6626 \times 10^{-5}$
\end{tabular}

De posse dos valores médios verdadeiros para as saídas dos sensores inerciais, utilizou-se (27) a (29) para se obter os valores médios verdadeiros para a orientação inicial do SINS, isto é, os ângulos de Euler que mais se aproximam do alinhamento real da plataforma, os quais, à semelhança das saídas dos sensores, são desconhecidos na prática. Os valores obtidos foram: $\theta=0.0553^{\circ}, \psi=$ $0.0656^{\circ}$ e $\phi=-0.3989^{\circ}$.

Após a determinação dos valores médios verdadeiros para as saídas dos sensores inerciais e para os ângulos de Euler, utilizou-se o desvio padrão de Allan para se estimar o randow walk angular do giroscópio apontado para o leste, obtendo-se: $r w_{y}=0.0058^{\circ} / \sqrt{h}$.

Finalmente, de modo a se se estimar o tempo de alinhamento em azimute necessário para se obter uma classe de precisão de $0.01^{\circ}$ no procedimento de auto-alinhamento (classe de precisão desejada para aplicações em veículos aeroespaciais), substituiu-se em (49), o valor do randow walk angular acima calculado, obtendo-se $T=5.78 \mathrm{~h}$. Este valor ficará evidente nos gráficos que serão apresentados a seguir.

\subsection{Análise do auto-alinhamento estacionário}

Uma vez determinados os valores médios verdadeiros para as saídas dos sensores inerciais e para o alinhamento do SINS, e estimado o tempo de alinhamento em azimute necessário para obtenção da classe de precisão desejada, procedeu-se com a análise propriamente dita do procedimento de auto-alinhamento apresentado na Seção 2.

Para fins de análise, dividiu-se a sequência de dados provenientes dos sensores inerciais em intervalos (tempos de alinhamento) de 1, 5, 10, 30 e 60 minutos, e para cada intervalo considerado, calculou-se os valores médios das forças específicas e velocidades angulares medidas. De posse de tais valores, utilizou-se (27) a (29) para se calcular, em cada intervalo, os correspondentes ângulos de Euler e a tendência estatística dos mesmos. Os resultados são apresentados nas Fig. 1 a 3 e nas Tabelas 2 e 3 .

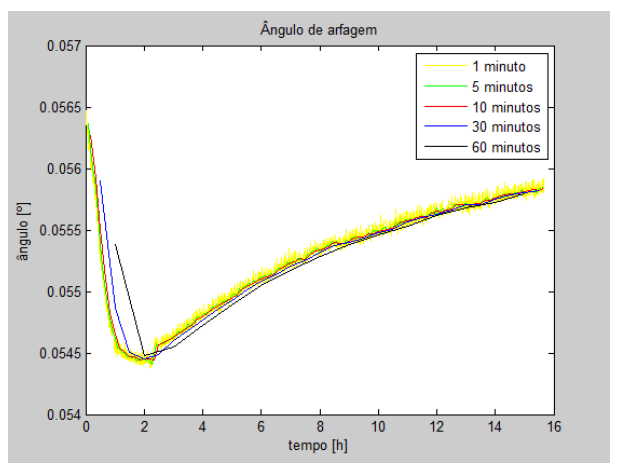

Figura 1: Ângulo de arfagem.

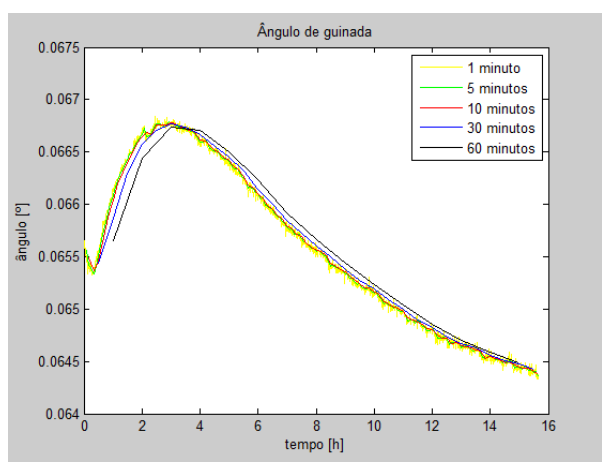

Figura 2: Ângulo de guinada.

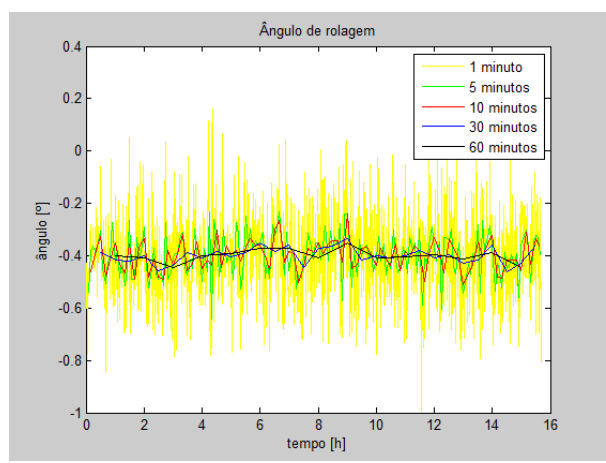

Figura 3: Ângulo de rolagem.

Tabela 2: Ângulos de Euler - valor médio.

\begin{tabular}{c|ccc}
$\mathrm{T}$ & $\theta\left[^{\circ}\right]$ & $\psi\left[^{\circ}\right]$ & $\phi\left[^{\circ}\right]$ \\
\hline $1 \mathrm{~min}$ & 0.0553 & 0.0656 & -0.3990 \\
$5 \mathrm{~min}$ & 0.0553 & 0.0656 & -0.3985 \\
$10 \mathrm{~min}$ & 0.0553 & 0.0656 & -0.3985 \\
$30 \mathrm{~min}$ & 0.0553 & 0.0656 & -0.3989 \\
$60 \mathrm{~min}$ & 0.0552 & 0.0656 & -0.4002
\end{tabular}

Tabela 3: Ângulos de Euler - desvio padrão.

\begin{tabular}{c|ccc}
$\mathrm{T}$ & $\theta\left[^{\circ}\right]$ & $\psi\left[^{\circ}\right]$ & $\phi\left[^{\circ}\right]$ \\
\hline $1 \mathrm{~min}$ & $4.4912 \times 10^{-4}$ & $7.8689 \times 10^{-4}$ & 0.1772 \\
$5 \mathrm{~min}$ & $4.4794 \times 10^{-4}$ & $7.8737 \times 10^{-4}$ & 0.0803 \\
$10 \mathrm{~min}$ & $4.4845 \times 10^{-4}$ & $7.8921 \times 10^{-4}$ & 0.0555 \\
$30 \mathrm{~min}$ & $4.4668 \times 10^{-4}$ & $7.9115 \times 10^{-4}$ & 0.0309 \\
$60 \mathrm{~min}$ & $4.2667 \times 10^{-4}$ & $7.8415 \times 10^{-4}$ & 0.0257
\end{tabular}


Analisando a Tabela 3, pode-se observar que para a obtenção de uma classe de precisão de $0.01^{\circ}$ na estimativa dos ângulos de arfagem e guinada, não é necessário mais do que 1 minuto (ou menos) de amostragem. Pode-se observar, também, que o desvio padrão da estimativa destes ângulos praticamente não varia, o que demonstra que a precisão máxima destas estimativas já foi alcançada, e o aumento no tempo de alinhamento não resultará em grande melhoria.

Por outro lado, para o ângulo de rolagem (alinhamento em azimute), observa-se que o desvio padrão calculado diminui à medida que o tempo de alinhamento aumenta, indicando que a máxima precisão para o alinhamento em azimute ainda não foi ancançada. Analisando o desvio padrão após 60 minutos de amostragem, evidencia-se que a média acumulada das leituras dos sensores ainda propaga uma incerteza da ordem de $0.0257^{\circ}$, quase três vezes maior que a desejada $\left(0.01^{\circ}\right)$.

Para se avaliar o tempo de alinhamento necessário para se obter a classe de precisão de $0.01^{\circ}$ no alinhamento em azimute, considerou-se a média acumulada das leituras dos sensores não apenas nos instantes $1,5,10,30$ e 60 minutos, mas desde primeiro instante de amostragem até o último. Traçou-se, em seguida, gráficos da evolução das estimativas do ângulo de rolagem em função do tempo de alinhamento. Os resultados são mostrados na Fig. 4.

Analisando a Fig. 4 observa-se primeiramente que, como esperado, as estimativas do ângulo de rolagem convergem para os respectivo valor médio verdadeiro, calculado na Seção 4.1. Via análise gráfica, pôde-se observar também, que a precisão desejada para este ângulo, só é alcançada depois de aproximadamente 6 horas de amostragem. Este valor condiz com o tempo de alinhamento em azimute calculado em (49), e demonstra que, de fato, as incertezas provenientes do giroscópio apontado para leste constituem o fator preponderante na determinação do tempo de alinhamento em azimute, que por sua vez, determina o tempo de alinhamento total de um SINS.

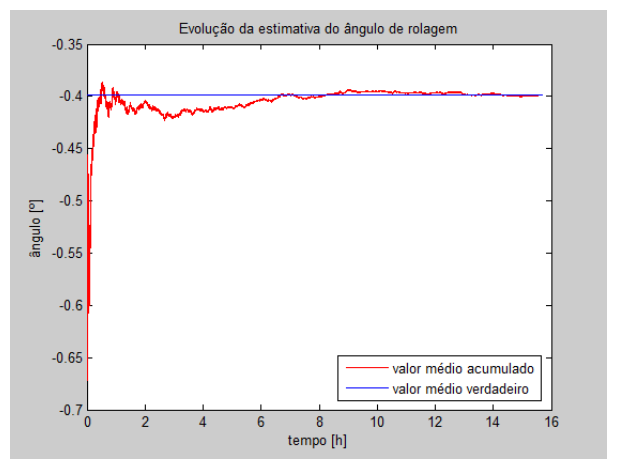

Figura 4: Evolução da estimativa do ângulo de rolagem.

\section{Conclusões}

Neste artigo, foi demonstrado experimentalmente que o tempo total de auto-alinhamento de um SINS é determinado pelo alinhamento em azimute, o qual por sua vez, é influenciado principalmente pelo randow noise do giroscópio apontado para o leste. Para o caso específico deste ensaio, o tempo de alinhamento necessário para se obter a classe de precisão de $0.01^{\circ}$ no procedimento de autoalinhamento foi de aproximadamente 5,78 horas.

Por fim, como sugestão para trabalhos futuros, os autores propõem o estudo da implementação de um algoritmo baseado em filtragem estocástica e estimação ótima, subsequente ao procedimento de auto-alinhamento apresentado neste artigo, o qual figura como solução mais viável para a diminuição do tempo de alinhamento necessário para se obter a classe de precisão desejada.

\section{Agradecimentos}

Os autores gostariam de agradecer à Fundep, Fundação de Desenvolvimento da Pesquisa, pelo apoio financeiro.

\section{Referências}

Britting, K. R. (1971). Inertial Navigation Systems Analysis, John Wiley \& Sons, Inc.

Farrel, J. and Barth, M. (1999). The Global Positioning System \& Inertial Navigation, McGraw-Hill Companies, Inc.

Han, S., Wang, J. and Knight, N. (2009). Using Allan variance to determine the calibration model of inertial systems for GPS/INS integration, 6th International Symposium on $\mathrm{Mo-}$ bile Mapping Technology.

Jekeli, C. (2000). Inertial Navigation Systems with Geodetic Applications, Walter de Gruyter $\mathrm{GmbH} \& \mathrm{Co}$.

Jiang, Y. F. (1998). Error analysis of analytic coarse alignment methods, IEEE Transactions on Aerospace and Electronic Systems $\quad 34(1)$ : $\quad 334-337 . \quad$ DOI: $10.1109 / 7.640292$

Pio, R. L. (1966). Euler angle transformations, IEEE Transactions on Automatic Con-trol 11(4): 707-715. DOI: 10.1109/TAC.1966.1098430

Savage, P. G. (2007). Strapdown Analytics, Strapdown Associates, Inc.

Titterton, D. H. and Weston, J. L. (2004). Strapdown Inertial Navigation Technology, Institution of Electrical Engineers.

Vuolo, J. H. (1992). Fundamentos da Teoria de Erros, Editora Edgard Blücher Ltda. 